\title{
Carnosine improves diabetic retinopathy via the MAPK/ERK pathway
}

\author{
YONG GUO, CHENJUN GUO, WENJING HA and ZHENHUA DING
}

Department of Ophthalmology, Tangdu Hospital, Air Force Medical University, Xi'an, Shaanxi 710038, P.R. China

Received May 8, 2018; Accepted November 28, 2018

DOI: $10.3892 /$ etm.2019.7223

\begin{abstract}
Diabetic retinopathy (DR) is one of the most common causes of blindness in developed countries. Due to its asymptomatic onset and progressive disease course, DR is typically diagnosed at a late stage when treatment options are limited and therefore often results in irreversible blindness. Studies have demonstrated that carnosine may prevent and treat DR. In a previous study, the positive effect of carnosine on DR was determined and it was revealed that there may be an association between carnosine and the mitogen-activated protein kinase (MAPK)/extracellular signal related kinase (ERK) signaling pathway. To assess the interaction between carnosine and the MAPK/ERK signaling pathway, changes in PKC, ERK and p-ERK expression was assessed in diabetic rats following treatment with carnosine, PD98059 or U46619 via reverse transcription-quantitative polymerase chain reaction and western blotting. The results demonstrated that the expression of ERK and p-ERK were significantly suppressed following treatment with carnosine, but no significant effect on the expression of PKC was identified, which indicates that suppressing the activation of the MAPK/ERK signaling pathway may serve an important role in carnosine-induced DR prevention and treatment.
\end{abstract}

\section{Introduction}

Diabetes mellitus (DM) is a systemic metabolic disorder characterized by impaired glucose metabolism, the incidence of which continues to grow at an alarming rate from $10.6 \%$ in 1989 to $32.1 \%$ in 2009 in Saudi Arabia based (1) A severe microvascular complication of DM, diabetic retinopathy (DR), is one of the four major blinding diseases that has become the leading cause of adult blindness, according to surveys

Correspondence to: Dr Yong Guo, Department of Ophthalmology, Tangdu Hospital, Air Force Medical University, 569 Xinsi Road, Xi'an, Shaanxi 710038, P.R. China

E-mail: guoyongeye@163.com

Key words: diabetic retinopathy, diabetic retinopathy rats, carnosine, mitogen-activated protein kinase/extracellular signal-related kinase signaling pathway conducted in other developed countries, including the United States and Japan (2). DR is typically diagnosed at a late stage when treatment options are limited. Due to its asymptomatic onset and progressive disease course, DR often results in irreversible blindness, which has a shortage of effective treatment due to the complex nature of the disease and limited knowledge of its pathogenic mechanism (3-5). Therefore, finding an effective treatment for DR has important clinical significance.

Poor blood glucose control, hypertension, hyperlipidemia and obesity may be involved in the development of DR, and may also contribute to the development of neuropathy (6). Studies $(7,8)$ assessing the pathogenetic pathways that lead to microangiopathy indicate that many genes may be involved in its development. Known biochemical mechanisms that underlie DR include advanced glycation end-product (AGE) formation, oxidative stress, the activation of protein kinase $\mathrm{C}$ (PKC) isoforms and polyol and hexosamine pathway activity (7). As a result of impaired pathway activity, a number of issues may arise, including retinal tissue hypoxia, endothelial cell dysfunction, vasodilation impairment, angiogenic factor hyperactivation and changes to the extracellular matrix (7). At present, the treatment of DR primarily targets its proliferation, but the restoration of lost visual function remains difficult (8).

Carnosine is an endogenous dipeptide consisting of beta-alanine and L-histidine that is produced in skeletal muscle and the nervous system, which is influenced by diet $(9,10)$. It has been reported that many biological effects of carnosine, including anti-inflammation, anti-aging, immune-regulation and anti-diabetes are associated with its anti-oxidative activities (11-13). Carnosine has been revealed to be an effective scavenger for the removal of reactive carbonyl species, as determined in a mouse model of diabetic nephropathy, and the reactive species located in pancreatic $\beta$-cells $(10,14)$. Javadi et al (15) demonstrated that carnosine primarily prevents dehydroascorbic acid-induced unfolding and the aggregation of lens proteins and significant lens opacity. Furthermore, other studies have revealed that carnosine exerts a positive effect on the prevention and treatment of DR, but it has no association with anti-oxidation and anti-glycosylation $(16,17)$.

Mitogen-activated protein kinase (MAPK) is a signal transduction pathway that is involved in various physiological processes, including gene expression and the proliferation, differentiation, death and survival of various cells $(18,19)$. Members of the MAPK family are regulated by a cascade 
of phosphorylation and are activated by extracellular stimulus (20). In the process of ERK1/2 phosphorylation, the extracellular signal related kinase 1/2 (ERK1/2) cascade is involved in the MAPK pathway, while MAPK signaling is activated by MAPK kinase 1 (21). The activation of the MAPK/ERK signaling pathway has been used frequently for the study of diabetic complications (22). Previous studies have also revealed that the PKC signaling pathway is involved in diabetic wound healing (23) and diabetic myocardial injury (24). The current study assessed the alteration of PKC, ERK, and phosphorylated (p)-ERK expression in diabetic rats following treatment with carnosine, PD98059 (an inhibitor of MAPK/ERK) or U46619 (an activator of MAPK/ERK). Reverse transcription-quantitative polymerase chain reaction (RT-qPCR) and western blotting was performed to assess the association between carnosine and the MAPK/ERK signaling pathway. The results revealed that suppressing the activation of the MAPK/ERK signaling pathway may serve an important role in the prevention and treatment carnosine-induced DR. However, the PKC pathway may not be involved in this process.

\section{Materials and methods}

Reagents and animals. Streptozotocin (STZ) was purchased from Sigma-Aldrich (Merck KGaA; Darmstadt, Germany). Cluster of differentiation (CD)31 antibodies were purchased from Abcam (Cambridge, UK), and Goat anti-Mouse Immunoglobulin $\mathrm{G}(\mathrm{IgG}) \mathrm{H} \& \mathrm{~L}$-cyanine 3 (Cy3) antibodies were purchased from ProteinTech Group, Inc. (Chicago, IL, USA). A total of 25 male sprague dawley rats (age, 7 weeks; weight, 180-185 g) were purchased from the Experimental Animal Center of Southern Medical University (Guangzhou, China). All rats were housed in a specific pathogen free room at a temperature of $25^{\circ} \mathrm{C}$ and a humidity of $50 \%$ under a $12 \mathrm{~h}$ light/dark cycle, with ad libitum access to food and water.

Ethics statement. All animal experiments were performed in accordance with the recommendations included in the Guide for the Care and Use of Laboratory Animals of the National Institutes of Health. Furthermore, the protocol of the current study was approved by the Committee on the Ethics of Animal Experiments of Tangdu Hospital, Air Force Medical University (Xi'an, China).

Establishment of diabetic rat model. Following a week of feeding, the body weight of rats was measured. A total of 10 rats were randomly divided into 2 groups: The DM group $(\mathrm{n}=5$; weight, 200-220 g) and the normal group ( $\mathrm{n}=5$; weight, 180-200 g). Rats in the DM group were intraperitoneally injected once with $2 \% \mathrm{STZ}$ at a dose of $60 \mathrm{mg} / \mathrm{kg}$. Animals were considered diabetic when glucose levels were $>16 \mathrm{mM}$ at $72 \mathrm{~h}$ following injection. Normal rats were administered the same quantity $(100 \mu \mathrm{l})$ of citrate buffer solution $(0.02 \mathrm{~mol} / \mathrm{l}$; $\mathrm{pH}=4.5$ ). Rats were euthanized via an intraperitoneal injection of $240 \mathrm{mg} / \mathrm{kg}$ sodium pentobarbital followed by cervical dislocation. Retinas were then harvested for cell isolation.

Rat retinal vascular endothelial cell (RVEC) isolation and cell culture. To establish a primary cell culture of rat RVECs, retinas isolated from rats of each group were digested with trypsin at $37^{\circ} \mathrm{C}$ for $30 \mathrm{~min}$. Residual retinas were then minced and fragments were filtered through a $100 \mu \mathrm{m}$ cell strainer. Following harvest, fragments from the strainer were incubated in $3 \mathrm{ml}$ of mixed collagenase (Sigma-Aldrich) for $30 \mathrm{~min}$ at room temperature. Digestion was stopped using complete medium (Dulbecco's Modified Eagles medium supplemented with $10 \%$ fetal bovine serum, $100 \mathrm{U} / \mathrm{ml}$ penicillin and $100 \mu \mathrm{g} / \mathrm{ml}$ streptomycin, all Thermo Fisher Scientific, Inc., Waltham, MA, USA) at room temperature. Following centrifugation at $252 \mathrm{x} \mathrm{g}$ at room temperature for $5 \mathrm{~min}$, cells were suspended in complete medium. The above suspension was transferred to a culture flask precoated with $1 \%$ fibronectin (Sigma-Aldrich) and cultured at $37^{\circ} \mathrm{C}$ in a $5 \% \mathrm{CO}_{2}$ humidified incubator. The medium was changed every 2.5 days until all cells formed confluent monolayers. Cells were passaged at a split ratio of $1: 2$.

Cell identification. Rat RVECs were seeded $\left(7 \times 10^{3}\right.$ cells $)$ in 12-well plates containing coverslips for $24 \mathrm{~h}$ at $37^{\circ} \mathrm{C}$ and then fixed using $4 \%$ paraformaldehyde for $10 \mathrm{~min}$ at room temperature and washed in triplicate with PBS. Samples were then blocked with normal goat serum (1:50; cat. no. ab7481; Abcam, Cambridge, UK) for $15 \mathrm{~min}$ at room temperature. Subsequently cell slides were incubated with primary antibodies (anti-CD31; cat. no. ab28364; 1:200; Abcam) at $37^{\circ} \mathrm{C}$ for $2 \mathrm{~h}$. Samples were washed with PBS in triplicate and probed with Goat anti-Mouse immunoglobulin G H\&L-Cy3 (cat. no. SA00009-1; 1:1,000; ProteinTech Group, Inc.) at $37^{\circ} \mathrm{C}$ for $1 \mathrm{~h}$. Hoechst 33258 (Beyotime Institute of Biotechnology, Haimen, China) was used to stain nuclei and images were captured using a fluorescence microscope (magnification, x50; Olympus Corporation, Tokyo, Japan) at room temperature for 15 min. Cell number analysis was performed using Image $\mathrm{J}$ 1.45 software (National Institutes of Health, Bethesda, MD) as described previously (25). The number of positive cells (with red coloration) and blue stained nuclei were counted separately. Positive rate was determined to be the number of positive red stained cells/the number of blue stained nuclei.

Drug treatment. Rat RVECs from each group were plated in 6-well plates containing $2 \mathrm{ml}$ of complete DMEM medium at a density of $2 \times 10^{5}$ cells/well. Following cell culture for $24 \mathrm{~h}$ at $37^{\circ} \mathrm{C}$, medium was exchanged for fresh complete DMEM supplemented with PD98059 (25 $\mu \mathrm{mol} / 1$; Merck KGaA), U46619 (80 $\mu \mathrm{mol} / \mathrm{l}$; Merck KGaA), PD98059 (25 $\mu \mathrm{mol} / \mathrm{l})$ in combination with carnosine (1\%; cat. no. C9625; Sigma-Aldrich; Merck KGaA), and U46619 (80 $\mu \mathrm{mol} / \mathrm{l})$ in combination with carnosine (1\%). The control group was treated with equal quantities of deionized solvent (DMSO or ethanol). The expression of PKC, ERK and p-ERK in each group was detected $24 \mathrm{~h}$ following treatment.

Retinal immunofluorescence staining. Of the 25 rats used in the current study, a total of 15 rats (weight, 235-245 g) were randomly divided into 3 groups ( $\mathrm{n}=5$ in each group): A Diabetes mellitus (DM) group, a DM+ carnosine $(100 \mathrm{mg} / \mathrm{kg} / \mathrm{d}$ by gavage for 10 days) group and a control group. Retinas were isolated from the DM group, the DM+ carnosine group and the control group, and fixed in $4 \%$ para-formaldehyde solution at room temperature for $15 \mathrm{~min}$. Retinas were subsequently 

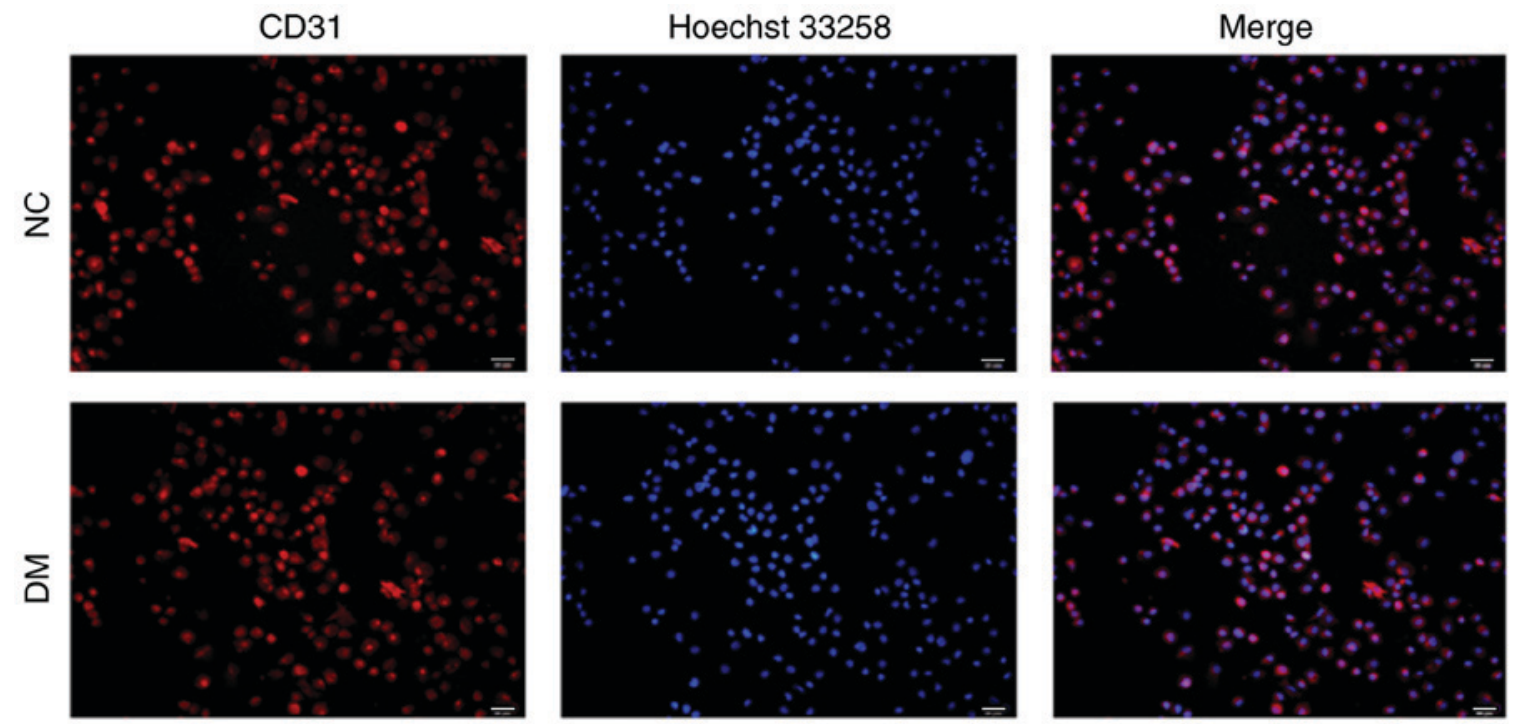

Figure 1. Representative immunofluorescence images of primary RVECs. Primary RVEC isolation and cultivation from normal or DM Rats were immunostained with anti-CD31 antibodies in vitro. Nuclei were stained with Hoechst 33258. Scale bar, $20 \mu \mathrm{m}$. RVEC, retinal vascular endothelial cells; CD, cluster of differentiation; NC, negative control; DM, diabetes mellitus.

sectioned (0.3 mm thick), stained with CD31 antibodies and Hochest as aforementioned and examined using fluorescence microscopy (magnification, x50; Olympus Corporation).

$R T-q P C R$. RT-qPCR analysis was performed as described previously (26). Total cellular RNA was extracted from rat retinas using a TRIzol reagent (Invitrogen; Thermo Fisher Scientific, Inc.) and cDNA was prepared using the M-MLV reverse transcriptase kit (Promega Corporation, Madison, WI) with random primers according to the manufacturer's protocol. The thermocycling conditions for PCR were as follows: Pre-denaturation for $10 \mathrm{~min}$ at $94^{\circ} \mathrm{C}$, denaturation for $20 \mathrm{sec}$ at $94^{\circ} \mathrm{C}$, annealing for $20 \mathrm{sec}$ at $55^{\circ} \mathrm{C}$, and extension for $20 \mathrm{sec}$ at $72^{\circ} \mathrm{C}$. Each PCR reaction was performed for 40 cycles. RT-qPCR analysis was performed using a Biosystems StepOne ${ }^{\mathrm{TM}}$ real-time PCR apparatus (Applied Biosystems; Thermo Fisher Scientific, Inc.) using standard procedures and analyzed using ABI Prism 7500 SDS software (Applied Biosystems; Thermo Fisher Scientific, Inc.). A Fast SYBR-Green Master Mix was obtained from Thermo Fisher Scientific, Inc. Data were presented as the relative expression following normalization to the expression of GAPDH using the $2^{-\Delta \Delta C q}$ method (27). The PCR primer sequences were as follows GAPDH forward, 5'-ACAGCAACAGGGTGG TGGAC-3' and reverse, 5'-TTTGAGGGTGCAGCGAAC TT-3'; PKC forward (5'-3'), 5'-TGGAGTCCTGCTGTATGA GATGTTG-3' and reverse, 5'-CGCTTTCCTGGGTGCTTG G-3'; ERK forward, 5'-GAAAGCATTACCTTGACCAG-3' and reverse, 5'-CTTTGGAGTCAGCATTTGG-3'.

Western blotting. The procedure for western blotting was performed as described previously (28). Total cellular protein was extracted using radioimmunoprecipitation assay lysis buffer containing phenylmethylsulfonyl fluoride Protease Inhibitor (Sigma-Aldrich; Merck KGaA). A BCA Protein Assay kit was utilized for protein determination (cat. no. 23225; Thermo Fisher Scientific). Proteins (30 $\mu \mathrm{g})$ were resolved in 5 or $12 \%$ SDS-PAGE gels and then transferred onto ployvinylidene difluoride (PVDF) membranes following electrophoresis. Subsequently, PVDF membranes were blocked using 5\% skimmed milk in TBST (20 mM Tris, pH 7.6, $150 \mathrm{mM}$ $\mathrm{NaCl}, 0.1 \%$ Tween-20; wt/vol) for $2 \mathrm{~h}$ at room temperature. Membranes were immunoblotted with Mouse anti-PKC (cat. no. P5704; 1:1,000; Sigma-Aldrich; Merck KGaA), ERK1/2 Rabbit Polyclonal (cat. no. 16443-1-AP; 1:1,000; ProteinTech Group, Inc.) and anti-p-ERK antibodies (cat. no. ab65142; 1:1,000; Abcam) at $4^{\circ} \mathrm{C}$ overnight. Samples were then incubated with IRDyeTM-800 conjugated anti-mouse (cat. no. P/N 925-32210; 1:10,000; Li-COR Biosciences, Lincoln, NE) and IRDye ${ }^{\circledR} 800 \mathrm{CW}$ Goat anti-Rabbit IgG $(\mathrm{H}+\mathrm{L})$ secondary antibodies (cat. no. P/N 925-32211; 1:10,000; Li-COR Biosciences) for $30 \mathrm{~min}$ at room temperature. The specific protein bands were detected using Odyssey Infrared Imaging System (Li-COR Biosciences) and analyzed with Image Studio ${ }^{\mathrm{TM}}$ Software 4.0 (Li-COR Biosciences). The quantification of each protein was normalized to $\beta$-actin.

Statistical analysis. All data were expressed as the mean \pm standard error of the mean and analyzed using GraphPad Prism5 software (GraphPad Software, Inc., La Jolla, CA). One-way analysis of variance followed by a Tukey's test was used to analyze the significant differences between groups. $\mathrm{P}<0.05$ was considered to indicate a statistically significant difference.

\section{Results}

RVEC determination. To determine whether the isolated and cultivated cells were RVECs, cells were fixed with $4 \%$ paraformaldehyde and subsequently stained with CD31, a specific marker of endothelial cells. As presented in Fig. 1,>90\% (Data not shown) of the isolated cells stained positive for CD31, thereby confirming that they were RVECs.

Carosine inhibited DM-caused retinal overvascularization. CD31 immunostaining revealed that the density of retinal 
A

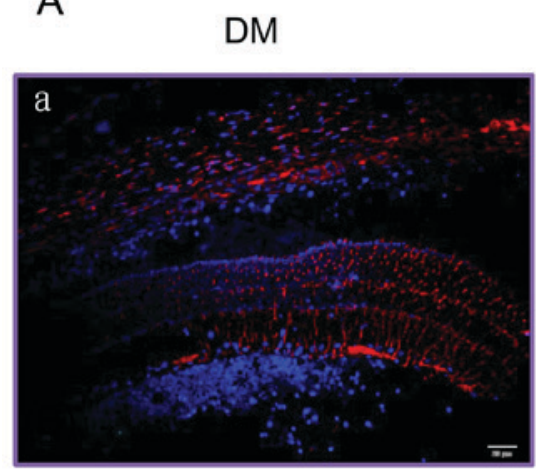

$\mathrm{DM}+$ Carnosine

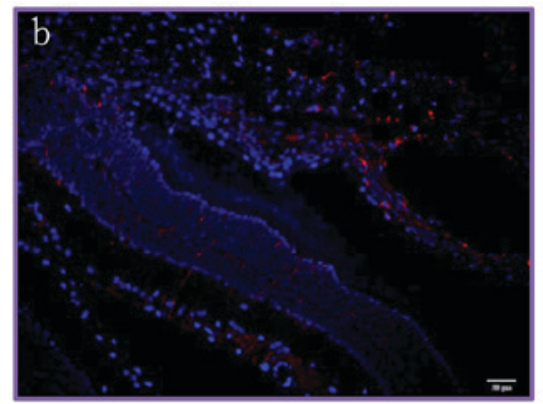

Control

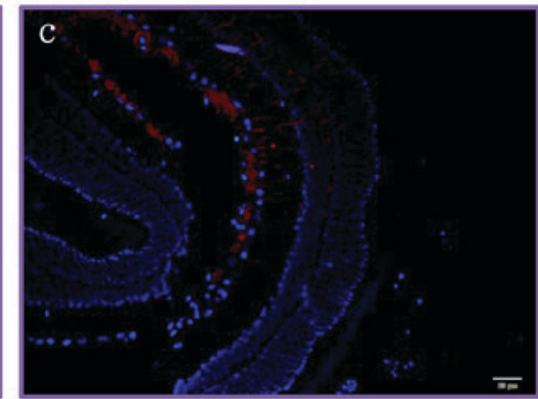

B

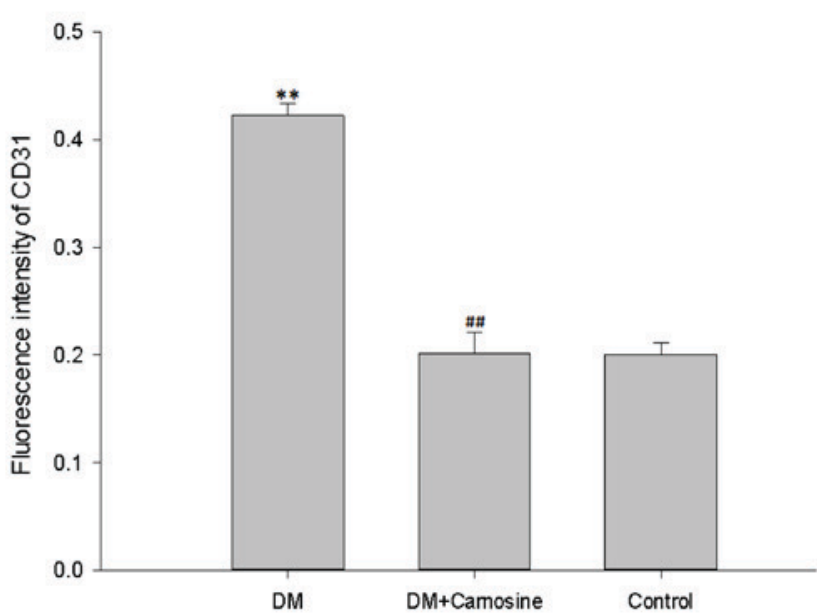

Figure 2. Representative immunofluorescence images of retinas obtained from rats. Immunofluorescence staining of retinas with CD31 antibodies in the DM group, DM+ carnosine group and Control group. Representative images of (Aa) DM rats at 9 weeks, (Ab) DM rats treated with carnosine at 9 weeks and (Ac) Control Sprague Dawley rats at 9 weeks. Red coloration indicates CD31 expression. Scale bar, $200 \mu \mathrm{m}$ (B) Quantitative results of CD31 expression. Data are presented as the mean \pm standard error of the mean $(n=3) .{ }^{* *} \mathrm{P}<0.01$ vs. Control rats, ${ }^{\# \#} \mathrm{P}<0.01 \mathrm{vs.} \mathrm{DM}$ rats. $\mathrm{CD}$, cluster of differentiation; DM, diabetes mellitus.

microvessels were significantly increased in DM rats compared with control rats and carosine markedly inhibited this increase of density in DM rats (Fig. 2).

The MAPK/ERK pathway is involved in the prevention and treatment of $D R$, as caused by carnosine. Changes of PKC, ERK and p-ERK expression in diabetic rats following treatment with carnosine, PD98059 and U46619 were assessed via RT-qPCR and western blotting. The mRNA levels of PKC (Fig. 3A) and ERK (Fig. 3B) of diabetic RVECs were significantly higher than those of normal RVECs. However, PKC mRNA levels in normal or diabetic RVECs were not substantially impacted by PD98059 and U46619 with or without carosine (Fig. 3A). Furthermore, ERK mRNA levels of RVECs was increased by U46619 and reduced by PD98059 and these changes were further enhanced by carosine (Fig. 3B).

The protein levels of PKC, ERK, and p-ERK in RVECs after treatment with PD98059, U46619, and carnosine were assessed via western blotting. Levels of PKC, ERK and phosphorylated ERK protein were significantly increased in diabetic RVECs compared with normal RVECs (Fig. 4A-D). PD98059 and U46619 increased the PKC protein level of normal RVECs but had little effect on the PKC protein level of diabetic RVECs. Furthermore, carosine inhibited PD98059 but augmented the U46619 induced increase of PKC. However,

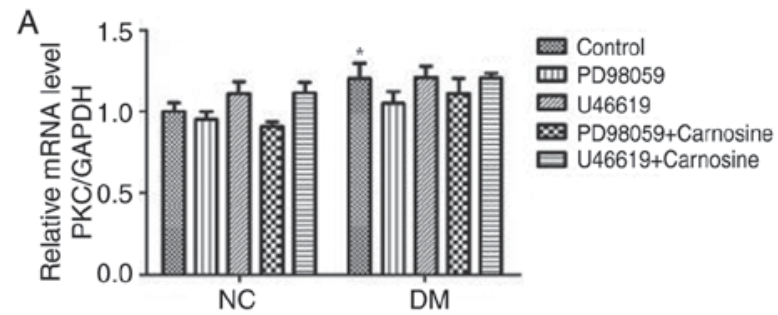

B

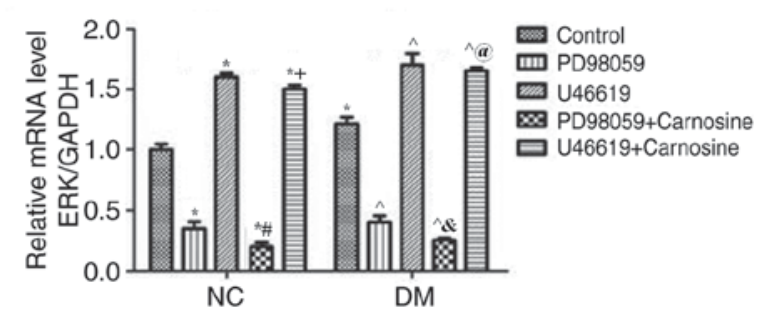

Figure 3. Carnosine modulates the expression of PKC/ERK mRNA in RVECs. The expression of (A) PKC and (B) ERK mRNA were determined via reverse transcription-quantitative polymerase chain reaction following treatment with or without PD98059, U46619 and carnosine. Expression was normalized to GAPDH and quantified. All measurements are presented as the mean \pm standard error of the mean from three independent experiments. " $\mathrm{P}<0.05$ vs. NC Control; ${ }^{\#} \mathrm{P}<0.05$ vs. NC PD98059; ${ }^{+} \mathrm{P}<0.05$ vs. U46619; ${ }^{\wedge} \mathrm{P}<0.05$ vs. $\mathrm{DM}$ Control; ${ }^{\circledR} \mathrm{P}<0.05$ vs. DM PD98059; ${ }^{\circledR} \mathrm{P}<0.05$ vs. DM U46619. PKC, protein kinase C; ERK, extracellular signal related kinase; RVEC, retinal vascular endothelial cells; NC, negative control; DM, diabetes mellitus. 
A

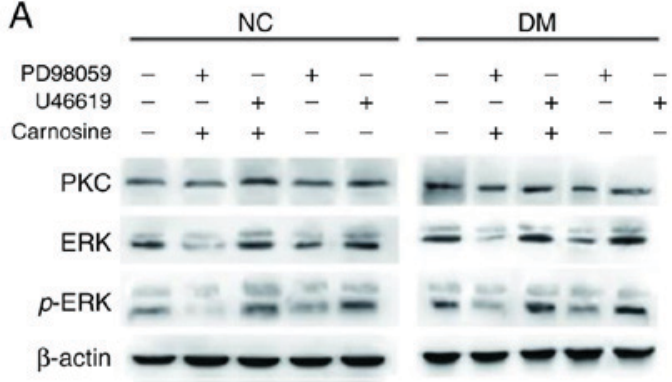

C

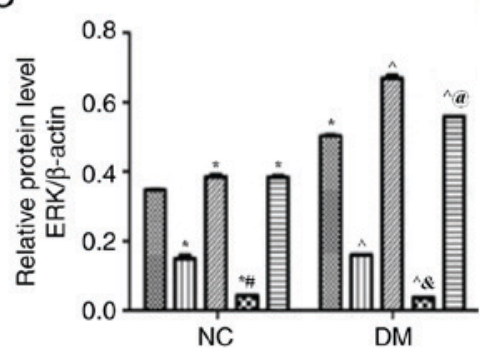

B

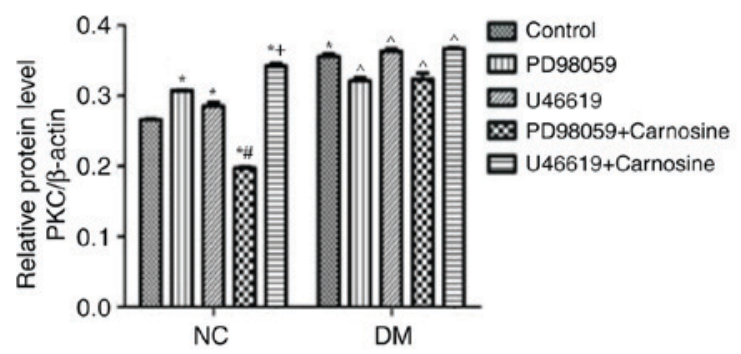

D

Figure 4. Carnosine regulates the level of PKC/ERK/p-ERK proteins in RVECs. (A) Western blotting determined the expression of PKC, ERK and p-ERK following treatment with or without PD98059, U46619 and carnosine for $24 \mathrm{~h}$ (A). The quantification of (B) PKC, (C) ERK, (D) p-ERK and (E) ERK/p-ERK expression was determined by normalizing to $\beta$-actin. Data are presented as the mean \pm standard error of the mean from 3 independent experiments. $\mathrm{P}<0.05 \mathrm{vs.} \mathrm{NC}$ Control; ${ }^{\text {P }}<0.05$ vs. NC PD98059; ${ }^{+} \mathrm{P}<0.05$ vs. U46619; ${ }^{\wedge} \mathrm{P}<0.05$ vs. DM Control; ${ }^{\star} \mathrm{P}<0.05$ vs. DM PD98059; ${ }^{\circledR} \mathrm{P}<0.05$ vs. DM U46619. PKC, protein kinase C; ERK, extracellular signal related kinase; p, phosphorylated; RVEC, retinal vascular endothelial cells; NC, negative control; DM, diabetes mellitus.

it did not effect the PKC levels of diabetic RVECs (Fig. 4A and B). ERK and phosphorylated ERK levels were reduced by PD98059 and increased by U46619 in normal and diabetic RVECs. Carosine treatment reduced ERK and phosphorylated ERK levels of normal and diabetic RVECs treated with PD98059 and U46619. Normal RVECs treated with U46619 however, were not affected (Fig. 4A, C and D). Taken together, these data suggest that the MAPK/ERK signaling pathway, rather than the PKC pathway, mediates carnosine-induced microvascular changes in DR.

\section{Discussion}

Carnosine ( $\beta$-Ala-1-His) is an imidazole dipeptide that has many biological functions. Numerous studies have demonstrated that the carnosine-carnosinase system serves a key role in the pathogenesis of diabetes and that carnosine may be used as a novel therapeutic agent against type 2 diabetes $(10,29,30)$. Cripps et al (10) revealed that following treatment with carnosine, insulin secretion from isolated mouse islets or insulinoma cell line $\beta$-cells was increased. They further revealed that the inhibition of insulin secretion caused by glucolipotoxic was reversed and that glucose uptake into skeletal muscle cells was enhanced. AGEs and oxidative stress serve a role in the development of diabetic complications (31). It has been demonstrated that carnosine can treat diabetic complications by decreasing oxidation and glycation products in the serum and livers of diabetic rats (30).

Visual impairment as a result of DR has a significant negative impact on patient quality of life and their ability to successfully manage their disease (32). Due to its asymptomatic onset and progressive disease course, identifying an effective treatment of DR is clinically significant. Pfister et al (33) determined that treatment with oral carnosine prevents vascular damage in experimental DR, independent of its biochemical function, which includes reactive oxygen species (ROS) or advanced glycation end (AGE) inhibition (33).

The MAPK/ERK signaling pathway has been determined to serve an important role in various pathogenetic pathways and physiological processes, including the regulation of cell cycle entry and proliferation, as well as in the development of DR by upregulating chronic inflammation (34). The MAPK/ERK signaling pathway includes various MAPKs, including ERKs, which integrate multiple biochemical and environmental signals (including Ras activation and the kinase cascade) via phosphorylation cascades (35). Gogg et al (36) demonstrated that the phosphorylation of ERK1/2 was significantly upregulated in Type 2 diabetes. A previous study revealed that after the degradation of the insulin receptor substrate (IRS)-1 protein, p-MAPK levels are upregulated following the increase of ERK1/2 phosphorylation in diabetes (37). However, the basic phosphorylation of ERK1/2 has also been demonstrated to be upregulated in adipose cells in type 2 diabetes (38). Additionally, IRS-1 has also been revealed to be decreased (39).

ERK is strongly activated in primary neuroretinal tissue and has been determined to be a neuroprotective regulator in the neuroretina, which is involved in the maintenance of the neuroretina- retinal pigment epithelium interaction $(40,41)$. It has been reported that the activation of ERK serves an important role in the maintenance of the inner blood-retinal barrier, similar to that of the RPE outer blood-retinal barrier (42). Van Dijk et al (43) demonstrated that the reversible suppression of ERK activity leads to disturbances in the neuroretina-RPE interaction and in the occurrence of SRF, which results in retinopathy. 
The glycation of proteins and the formation of AGEs serve an important role in DM and DR. An increase in AGE level has been identified in the retinas of patients with diabetes and has been determined to be positively correlated with the increase of blood glucose and the development of DR (44). Previous studies have demonstrated that the combination of $\mathrm{AGE}$ and the AGE receptor causes the activation of the MAPK/ERK signaling pathway, the activation of the oxidative stress response and the overexpression of certain cytokines (including inflammatory cytokines, lymphocyte adhesion molecules, vascular regulators and coagulants), which may lead to retinal lesions and vascular endothelial cell damage in vitro $(45,46)$. The current study revealed that the expression of $\mathrm{p}-\mathrm{ERK} / \mathrm{ERK}$ significantly changed following treatment with PD98059, U46619 and carnosine, respectively or in combination. The current study therefore hypothesized that the protective effect of carnosine in DR may be associated with AGEs and the MAPK/ERK signaling pathway. However, further studies are required to clarify how the interaction between carnosine and the MAPK/ERK signaling pathway improves DR.

A previous study has determined that the activity of $\mathrm{PKC}$, MEK and ERK1/2 is significantly increased in glomerular mesangial cells in STZ-induced and glucose-induced diabetic rats, and that the activation of MEK and ERK1/2 is PKC-dependent (46). The results of the current study revealed that there was almost no effect on the expression of PKC following treatment with PD98059, U46619 and carnosine, respectively or in combination, indicating that the suppression of the PKC-independent MAPK/ERK signaling pathway, rather than the PKC pathway, may serve an important role in the prevention and treatment of carnosine-induced DR.

In summary, the present study demonstrated that the MAPK/ERK signaling pathway may mediate carnosine-induced microvascular changes in DR.

\section{Acknowledgements}

Not applicable.

\section{Funding}

The current study was supported a grant obtained from the Innovation fund of the Air Force Medical University (grant no. 2014JCR010).

\section{Availability of data and materials}

The datasets used and/or analyzed during the current study are available from the corresponding author on reasonable request.

\section{Authors' contributions}

YG designed the current study, wrote the manuscript and established the diabetic rat model. CG performed western blotting and statistical analysis and RT-qPCR. WH performed retinal immunofluorescence staining, drug treatment and cell identification. ZD performed RVEC isolation and cell culture.

\section{Ethics approval and consent to participate}

All animal experiments were performed in accordance with the recommendations included in the Guide for the Care and Use of Laboratory Animals of the National Institutes of Health. Furthermore, the protocol of the current study was approved by the Committee on the Ethics of Animal Experiments of Tangdu Hospital, Air Force Medical University.

\section{Consent for publication}

Not applicable.

\section{Competing interests}

The authors declare that they have no competing interests.

\section{References}

1. Alotaibi A, Perry L, Gholizadeh L and Al-Ganmi A: Incidence and prevalence rates of diabetes mellitus in Saudi Arabia: An overview. J Epidemiol Glob Health 7: 211-218, 2017.

2. Mrozikiewicz-Rakowska B, Łukawska M, Nehring P, Szymański K, Sobczyk-Kopcioł A, Krzyżewska M, Maroszek P, Płoski R and Czupryniak L: Genetic predictors associated with diabetic retinopathy in patients with diabetic foot. Pol Arch Intern Med 128: 35-42, 2018.

3. Tan GS, Cheung N, Simó R, Cheung GC and Wong TY: Diabetic macular oedema. Lancet Diabetes Endocrinol 5: 143-155, 2017.

4. Tolentino MS, Tolentino AJ and Tolentino MJ: Current and investigational drugs for the treatment of diabetic retinopathy. Expert Opin Investig Drugs 25: 1011-1022, 2016.

5. Brownlee M, Aiello LP, Cooper ME, Vinik AI, Plutzky J and Boulton AJ: Complications of diabetes mellitus. In: Williams Textbook of Endocrinology (Thirteenth Edition) Elsevier, pp 1484-1581, 2017.

6. Happich M, Breitscheidel L, Meisinger C, Ulbig M, Falkenstein P, Benter $U$ and Watkins $\mathrm{J}$ : Cross-sectional analysis of adult diabetes type 1 and type 2 patients with diabetic microvascular complications from a German retrospective observational study. Curr Med Res Opin 23: 1367-1374, 2007.

7. Brownlee M: Biochemistry and molecular cell biology of diabetic complications. Nature 414: 813-820, 2001.

8. Ramasamy R, Vannucci SJ, Yan SS, Herold K, Yan SF and Schmidt AM: Advanced glycation end products and RAGE: A common thread in aging, diabetes, neurodegeneration, and inflammation. Glycobiology 15: 16R-28R, 2005.

9. Katakura Y, Totsuka M, Imabayashi E, Matsuda H and Hisatsune T: Anserine/carnosine supplementation suppresses the expression of the inflammatory chemokine CCL24 in peripheral blood mononuclear cells from elderly people. Nutrients 9: E1199, 2017.

10. Cripps MJ, Hanna K, Lavilla C Jr, Sayers SR, Caton PW, Sims C, De Girolamo L, Sale C and Turner MD: Carnosine scavenging of glucolipotoxic free radicals enhances insulin secretion and glucose uptake. Sci Rep 7: 13313, 2017.

11. Baye E, Ukropcova B, Ukropec J, Hipkiss A, Aldini G and de Courten B: Physiological and therapeutic effects of carnosine on cardiometabolic risk and disease. Amino Acids 48: 1131-1149, 2016.

12. Tan RR,Li YF,Zhang SJ,Huang WS, Tsoi B,Hu D, Wan X, Yang X, Wang Q, Kurihara $\mathrm{H}$ and He RR: Abnormal O-GlcNAcylation of Pax3 occurring from hyperglycemia-induced neural tube defects is ameliorated by carnosine but not folic acid in chicken embryos. Mol Neurobiol 54: 281-294, 2017.

13. Li YF, He RR, Tsoi B, Li XD, Li WX, Abe K and Kurihara H: Anti-stress effects of carnosine on restraint-evoked immunocompromise in mice through spleen lymphocyte number maintenance. PLoS One 7: e33190, 2012.

14. Albrecht T, Schilperoort M, Zhang S, Braun JD, Qiu J, Rodriguez A, Pastene DO, Krämer BK, Köppel H, Baelde H, et al: Carnosine attenuates the development of both type 2 diabetes and diabetic nephropathy in BTBR ob/ob mice. Sci Rep 7: 44492, 2017. 
15. Javadi S, Yousefi R, Hosseinkhani S, Tamaddon AM and Uversky VN: Protective effects of carnosine on dehydroascorbate-induced structural alteration and opacity of lens crystallins: Important implications of carnosine pleiotropic functions to combat cataractogenesis. J Biomol Struct Dyn 35: 1766-1784, 2017.

16. Klein BE: Overview of epidemiologic studies of diabetic retinopathy. Ophthalmic Epidemiol 14: 179-183, 2007.

17. Coleman SK, Rebalka IA, D'Souza DM and Hawke TJ: Skeletal muscle as a therapeutic target for delaying type 1 diabetic complications. World J Diabetes 6: 1323-1336, 2015.

18. Cargnello $M$ and Roux PP: Activation and function of the MAPKs and their substrates, the MAPK-activated protein kinases. Microbiol Mol Biol Rev 75: 50-83, 2011.

19. Pearson G, Robinson F, Beers Gibson T, Xu BE, Karandikar M, Berman K and Cobb MH: Mitogen-activated protein (MAP) kinase pathways: Regulation and physiological functions. Endocr Rev 22: 153-183, 2001.

20. Cervellini I, Galino J, Zhu N, Allen S, Birchmeier C and Bennett DL: Sustained MAPK/ERK activation in adult schwann cells impairs nerve repair. J Neurosci 38: 679-690, 2018.

21. Danquah A, de Zelicourt A, Colcombet J and Hirt H: The role of ABA and MAPK signaling pathways in plant abiotic stress responses. Biotechnol Adv 32: 40-52, 2014

22. Hirata K and Kubo K: Relationship between blood levels of $\mathrm{N}$-carboxymethyl-lysine and pentosidine and the severity of microangiopathy in type 2 diabetes. Endocr J 51: 537-544, 2004

23. Schaaf G, Dynowski M, Mousley CJ, Shah SD, Yuan P, Winklbauer EM, de Campos MK, Trettin K, Quinones MC, Smirnova TI, et al: Resurrection of a functional phosphatidylinositol transfer protein from a pseudo-Sec14 scaffold by directed evolution. Mol Biol Cell 22: 892-905, 2011

24. Maas M, Wang R, Paddock C, Kotamraju S, Kalyanaraman B, Newman PJ and Newman DK: Reactive oxygen species induce reversible PECAM-1 tyrosine phosphorylation and SHP-2 binding. Am J Physiol Heart Circ Physiol 285: H2336-H2344, 2003.

25. Grishagin IV: Automatic cell counting with ImageJ. Anal Biochem 473: 63-65, 2015

26. Mao L, Wang H, Ma F, Guo Z, He H, Zhou H and Wang N: Exposure to static magnetic fields increases insulin secretion in rat INS- 1 cells by activating the transcription of the insulin gene and up-regulating the expression of vesicle-secreted proteins. Int J Radiat Biol 93: 831-840, 2017.

27. Livak KJ and Schmittgen TD: Analysis of relative gene expression data using real-time quantitative PCR and the 2(-Delta Delta $\mathrm{C}(\mathrm{T}))$ method. Methods 25: 402-408, 2001.

28. Liao XH, Wang N, Liu QX, Qin T, Cao B, Cao DS and Zhang TC: Myocardin-related transcription factor-A induces cardiomyocyte hypertrophy. IUBMB Life 63: 54-61, 2011.

29. DeLisser HM, Newman PJ and Albelda SM: Molecular and functional aspects of PECAM-1/CD31. Immunol Today 15: 490-495, 1994.

30. Aydın AF, Bingül İ, Küçükgergin C, Doğan-Ekici I, Doğru Abbasoğlu S and Uysal M: Carnosine decreased oxidation and glycation products in serum and liver of high-fat diet and low-dose streptozotocin-induced diabetic rats. Int J Exp Pathol 98: 278-288, 2017.

31. Goldin A, Beckman JA, Schmidt AM and Creager MA: Advanced glycation end products: Sparking the development of diabetic vascular injury. Circulation 114: 597-605, 2006.

32. Hendrick AM, Gibson MV and Kulshreshtha A: Diabetic retinopathy. Prim Care 42: 451-464, 2015.

33. Pfister F, Riedl E, Wang Q, vom Hagen F, Deinzer M, Harmsen MC, Molema G, Yard B, Feng Y and Hammes HP Oral carnosine supplementation prevents vascular damage in experimental diabetic retinopathy. Cell Physiol Biochem 28 : 125-136, 2011.

34. Zhang L, Zhang ZK and Liang S: Epigallocatechin-3-gallate protects retinal vascular endothelial cells from high glucose stress in vitro via the MAPK/ERK-VEGF pathway. Genet Mol Res 15: 2016
35. Hu L, Zhang Y, Chen L, Zhou W, Wang Y and Wen J: MAPK and ERK polymorphisms are associated with PCOS risk in Chinese women. Oncotarget 8: 100261-100268, 2017.

36. Gogg S, Smith U and Jansson PA: Increased MAPK activation and impaired insulin signaling in subcutaneous microvascular endothelial cells in type 2 diabetes: The role of endothelin-1. Diabetes 58: 2238-2245, 2009.

37. Aguirre V, Werner ED, Giraud J, Lee YH, Shoelson SE and White MF: Phosphorylation of Ser307 in insulin receptor substrate-1 blocks interactions with the insulin receptor and inhibits insulin action. J Biol Chem 277: 1531-1537, 2002.

38. Carlson CJ, Koterski S, Sciotti RJ, Poccard GB and Rondinone CM: Enhanced basal activation of mitogen-activated protein kinases in adipocytes from type 2 diabetes: Potential role of p38 in the downregulation of GLUT4 expression. Diabetes 52: 634-641, 2003.

39. Rondinone CM, Wang LM, Lonnroth P, Wesslau C, Pierce JH and Smith U: Insulin receptor substrate (IRS) 1 is reduced and IRS-2 is the main docking protein for phosphatidylinositol 3-kinase in adipocytes from subjects with non-insulin-dependent diabetes mellitus. Proc Natl Acad Sci USA 94: 4171-4175, 1997.

40. Ulbrich F, Kaufmann KB, Coburn M, Lagrèze WA, Roesslein M, Biermann J, Buerkle H, Loop T and Goebel U: Neuroprotective effects of Argon are mediated via an ERK-1/2 dependent regulation of heme-oxygenase-1 in retinal ganglion cells. J Neurochem 134: 717-727, 2015.

41. Marra C, Gomes Moret D, de Souza Corrêa A, Chagas da Silva F, Moraes P, Linden R and Sholl-Franco A: Protein kinases JAK and ERK mediate protective effect of interleukin-2 upon ganglion cells of the developing rat retina. J Neuroimmunol 233: 120-126, 2011.

42. LoRusso PM, Krishnamurthi SS, Rinehart JJ, Nabell LM, Malburg L, Chapman PB, DePrimo SE, Bentivegna S, Wilner KD, Tan W and Ricart AD: Phase I pharmacokinetic and pharmacodynamic study of the oral MAPK/ERK kinase inhibitor PD-0325901 in patients with advanced cancers. Clin Cancer Res 16: 1924-1937, 2010.

43. van Dijk EH, Duits DE, Versluis M, Luyten GP, Bergen AA, Kapiteijn EW, de Lange MJ, Boon CJ and van der Velden PA: Loss of MAPK pathway activation in post-mitotic retinal cells as mechanism in MEK inhibition-related retinopathy in cancer patients. Medicine (Baltimore) 95: e3457, 2016.

44. Wautier JL and Guillausseau PJ: Advanced glycation end products, their receptors and diabetic angiopathy. Diabetes Metab 27: 535-542, 2001.

45. Genuth S, Sun W, Cleary P, Sell DR, Dahms W, Malone J, Sivitz W, Monnier VM and DCCT Skin Collagen Ancillary Study Group: Glycation and carboxymethyllysine levels in skin collagen predict the risk of future 10-year progression of diabetic retinopathy and nephropathy in the diabetes control and complications trial and epidemiology of diabetes interventions and complications participants with type 1 diabetes. Diabetes 54: 3103-3111, 2005.

46. Xu T, Wang NS, Fu LL, Ye CY, Yu SQ and Mei CL: Celecoxib inhibits growth of human autosomal dominant polycystic kidney cyst-lining epithelial cells through the VEGF/Raf/MAPK/ERK signaling pathway. Mol Biol Rep 39: 7743-7753, 2012.

This work is licensed under a Creative Commons Attribution-NonCommercial-NoDerivatives 4.0 International (CC BY-NC-ND 4.0) License. 